\title{
Discussion of 014405JOR by L. C. Hsiao et al.
}

Citation: Journal of Rheology 58, 1505 (2014); doi: 10.1122/1.4892818

View online: https://doi.org/10.1122/1.4892818

View Table of Contents: http://sor.scitation.org/toc/jor/58/5

Published by the The Society of Rheology

\section{Articles you may be interested in}

Two-step yielding in magnetorheology

Journal of Rheology 58, 1507 (2014); 10.1122/1.4880675

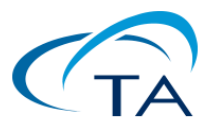

The WORLD'S most

VERSATILE platform for RHEOLOGICAL MEASUREMENTS

The Discovery Hybrid Rheometer
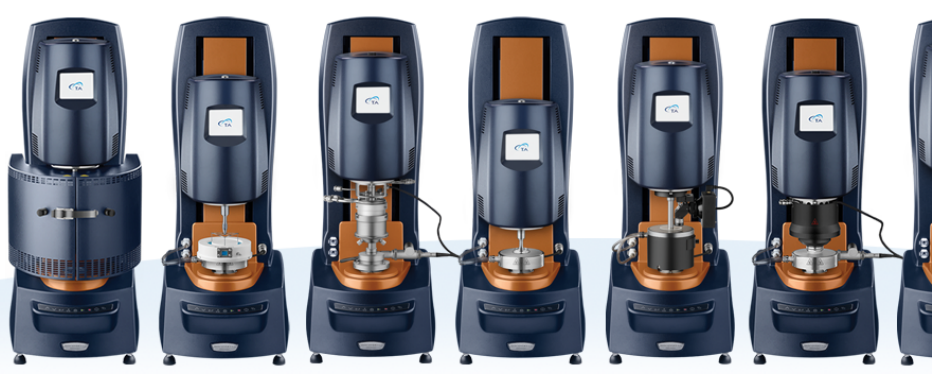


\section{Discussion of 014405JOR by L. C. Hsiao et al.}

Question: Emanuela Del Gado: Would it be possible to use this system and experimental setup to obtain a spatial map of possible mechanical heterogeneities in the gel, especially in connection with different morphologies (from stringy to a more thick gel)?

Author Response: A map of mechanical heterogeneities in a gel would be an interesting use of the new experimental system. One could imagine that this type of spatial map may need to be done through a series of experiments. First, imaging the structure of the gels would determine what type of structural elements (single strands, clusters, and dense particle networks) is present within the gels. Characterization of the mechanical properties of those structures may need to be done separately by recreating similar structures with the optical tweezers and then using the tweezers to test their mechanical properties. This could include studying the bending mechanics of strands or inducing cluster breakup and rearrangement. The tweezer experiments could provide insight into how the individual structural components would behave in the bulk gel. Alternatively, it may be possible to include additional probe particles of a higher refractive index contrast (such as melamine particles) to directly probe specific regions in the gels, similar to the earlier work of Lee and Furst (2008).

Question: Marco Laurati: Due to the significant mismatch in refractive index between particles and solvent in the system you described, how far from the coverslip can one acquire volume scans, or in other words, can one acquire data really in the bulk of the system, i.e., far enough from the coverslips in order to avoid wall effects?

Author Response: In the model system $\left(w_{\mathrm{CH}}=0.37\right)$ with a refractive index contrast, $n_{\mathrm{ct}}=n_{\mathrm{p}} / n_{\mathrm{s}}=1.029$, we capture image volumes at a distance of $\geq 10 \mu \mathrm{m}$ from the coverslip. (Particles can be observed and identified up to $\sim 50 \mu \mathrm{m}$ above the coverslip in this system.) Because the distance at which these image volumes are captured is at least 8 times the particle diameter from the coverslip $(2 a=1.15 \mu \mathrm{m})$, they can be considered as a representative of the structure of the bulk sample. Similarly, wall effects within the optical trapping experiments are avoided during the rupture force measurements by using the laser tweezers to move the particles to the middle of the sample away from both the top and bottom surfaces.

\section{Reference}

Lee, M. H., and E. M. Furst, "Response of a colloidal gel to a microscopic oscillatory strain," Phys. Rev. E 77, 041408 (2008). 